Tibault Reveyrand

email : tibault.reveyrand@laposte.net web : http://membres.lycos.fr/treveyrand

\title{
A Time Domain Envelope Vectorial Network Analyzer for Non-linear Measurement Based Modeling Accounting Impedance Mismatches
}

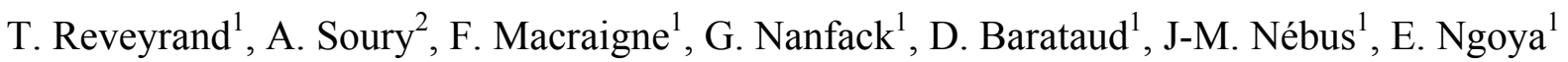
${ }^{1}$ XLIM-UMR CNRS 6172 - 123 Avenue Albert Thomas, 87060 Limoges Cedex, France

${ }^{2}$ Xpedion Design Systems - 24 rue Atlantis - Ester Technopole, 87280 Limoges, France

2006 IEEE INSTRUMENTATION AND MEASUREMENT TECHNOLOGY CONFERENCE SORRENTO, ITALIA 24 - 27 APRIL 2006

(C) 2006 IEEE. Personal use of this material is permitted. However, permission to reprint/republish this material for advertising or promotional purposes or for creating new collective works for resale or redistribution to servers or lists, or to reuse any copyrighted component of this work in other works must be obtained from the IEEE. 


\title{
A Time Domain Envelope Vectorial Network Analyzer for Non-linear Measurement Based Modeling Accounting Impedance Mismatches
}

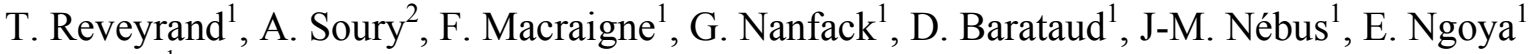 \\ ${ }^{1}$ XLIM-UMR CNRS 6172 - 123 Avenue Albert Thomas, 87060 Limoges Cedex, France \\ ${ }^{2}$ Xpedion Design Systems - 24 rue Atlantis - Ester Technopole, 87280 Limoges, France
}

\begin{abstract}
This paper presents a measurement setup which enables power amplifier characterization driven by radio frequency modulated signals. Incident and reflected absolutes waves at both ports of the device under test can be measured even in a source pull or load pull environment. The structure and the calibration procedure of the setup are explained. Measurements examples are shown and then used in order to generate a new kind of complex envelope behavioral model. This model is taking into account both high frequency memory effect and loading impedance mismatch.
\end{abstract}

Keywords - Nonlinear scattering parameters, power amplifier measurements, sytem systems, modelling.

\section{INTRODUCTION}

In the last years, many advances have been made both in characterization and device modeling for simulation. Those advances had imply the use of time domain analysis even in characterization. So, Microwave Transient Analyzer or LSNA are commonly used in order to analyze a nonlinear device presenting RF fundamental and harmonics frequencies. Concerning the characterization of device for communication where the fundamental is modulated with an large bandwidth arbitrary signal and the harmonics are not considered, those instruments imply some limitations (the frequency bandwidth). A measurement setup was presented in [1]. This bench was well suited for system level simulation and modeling, but assumed that the device under test will be used in a fifty ohms environment. This paper presents an enhancement of this setup and allow vectorial measurement around the RF fundamental : the four waves a1, b1, a2 and b2 can be extracted simultaneously and independently in a large bandwidth.

\section{MEASUREMENT SET-UP DESCRIPTION}

A block diagram of the measurement setup in given figure 1 (L-S band). The bench includes an emitter part and a 4 channels receiver part.

The emitter part generates a FI modulated signal with an arbitrary waveform generator (12 bits - $250 \mathrm{MS} / \mathrm{s})$ and transpose it to L or S band with an IQ modulator. Spurious frequencies at the reference plane vanish by the use of a local oscillator rejection loop and a band pass filter. A linear amplifier and a step attenuator fit the power range at the reference plane.

The receiver part measures simultaneously the IF image of the 4 waves (a1 b1 a2 and b2) located at the reference plane.
The frequency translation is done with four mixer. The use of four step attenuator optimize the linearity of the mixers and the dynamic of the final detector : a four channel sampling oscilloscope.

A tuner has been added to this original setup in order to extract nonlinear S parameters [8] [9] of a band S amplifier.

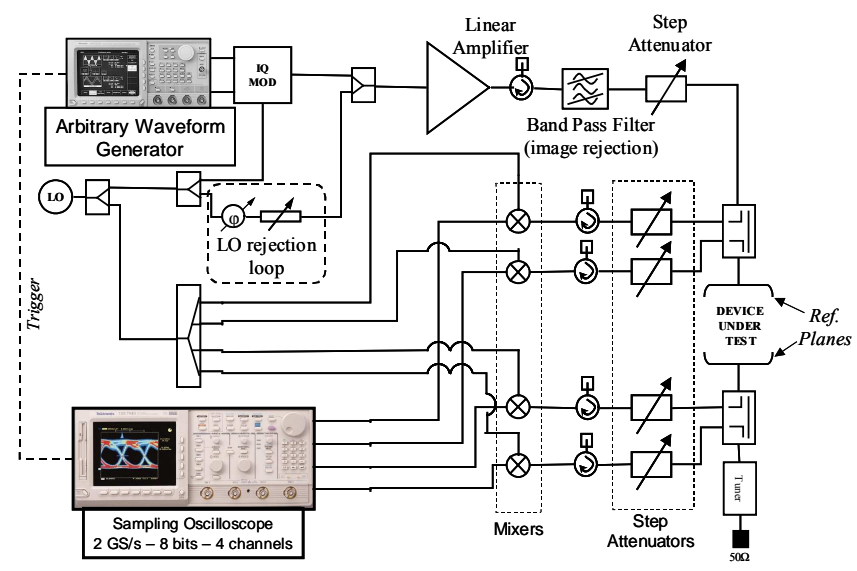

Fig. 1 : Schematic of the time domain envelope VNA

\section{CALIBRATION PROCEDURE}

The calibration procedure of the receiver part is divided in two steps.

First of all, we carry out a vectorial relative calibration of a four channel system as explained in [2]. An eight error term model is sufficient because of our system measure simultaneously the four waves. Therefore we can apply 12 terms-SOLT method [3] or a least-square 8 terms SOLT method for connectorized device. LRM [2], LRRM [4], or TRL [5] methods are much more suitable for on-wafer devices. The 8 terms error model lead us to the equation (1) : the relation between raw measured data $\left(a_{0}, b_{0}, a_{3}\right.$ and $\left.b_{3}\right)$ and waves in the reference planes $\left(a_{1}, b_{1}, a_{2}\right.$ and $\left.b_{2}\right)$.

$$
\left(\begin{array}{l}
a_{1} \\
b_{1} \\
a_{2} \\
b_{2}
\end{array}\right)=\left[\begin{array}{cccc}
1 & \beta_{1} & 0 & 0 \\
\gamma_{1} & \delta_{1} & 0 & 0 \\
0 & 0 & \alpha_{2} & \beta_{2} \\
0 & 0 & \gamma_{2} & \delta_{2}
\end{array}\right] \cdot\left(\begin{array}{l}
a_{0} \\
b_{0} \\
a_{3} \\
b_{3}
\end{array}\right)
$$

The linear system may be written $[\mathrm{A}] .(\mathrm{X})=(\mathrm{B})$ where $(\mathrm{X})$ is the 7 error terms vector as shown on figure 2 . 
Thus comes $(\mathrm{X})=\left[[\mathrm{A}]^{\prime} \cdot[\mathrm{A}]\right]-1 .[\mathrm{A}]^{\prime}$.(B) with $[\mathrm{A}]^{\prime}$, the conjugate transpose of $[\mathrm{A}]$.

\begin{tabular}{|c|c|c|c|c|c|c|c|c|c|}
\hline$-\mathrm{L} 1 . \mathrm{b}_{0}$ & $a_{0}$ & $\mathrm{~b}_{0}$ & 0 & 0 & 0 & 0 & & {$\left[\mathrm{~L} 1 . \mathrm{a}_{0}\right]$} & Load - Port 1 - Forward \\
\hline$-\mathrm{Ol}_{1} \mathrm{~b}_{0}$ & $a_{0}$ & $b_{0}$ & 0 & 0 & 0 & 0 & & $\mathrm{O}_{1 . \mathrm{a}_{0}}$ & Open - Port 1 - Forward \\
\hline$-S 1 . b_{0}$ & $a_{0}$ & $\mathrm{~b}_{0}$ & 0 & 0 & 0 & 0 & & $\mathrm{~S} 1 . \mathrm{a}_{0}$ & Short - Port 1 - Forward \\
\hline 0 & 0 & 0 & $-\mathrm{L} 2 . \mathrm{a}_{3}$ & $-\mathrm{L} 2 . \mathrm{b}_{3}$ & $a_{3}$ & $b_{3}$ & $\gamma_{1}$ & 0 & Load - Port 2 - Reverse \\
\hline 0 & 0 & 0 & $-02 . a_{3}$ & $-02 . b_{3}$ & $a_{3}$ & $b_{3}$ & $\alpha$ & 0 & Open - Port 2 - Reverse \\
\hline 0 & 0 & 0 & $-\mathrm{S} 2 . \mathrm{a}_{3}$ & $-\mathrm{S} 2 \cdot \mathrm{b}_{3}$ & $a_{3}$ & $b_{3}$ & $\alpha_{2}$ & 0 & Short - Port 2 - Reverse \\
\hline $\mathrm{b}_{0}$ & 0 & 0 & 0 & 0 & $-a_{3}$ & $-b_{3}$ & $\beta_{2}$ & $-\mathrm{a}_{0}$ & Thru (1) - Forward \\
\hline $\mathrm{b}_{0}$ & 0 & 0 & 0 & 0 & $-a_{3}$ & $-b_{3}$ & & $-\mathrm{a}_{0}$ & Thru (1) - Reverse \\
\hline 0 & $a_{0}$ & $\mathrm{~b}_{0}$ & $-a_{3}$ & $-b_{3}$ & 0 & 0 & & 0 & Thru (2) - Forward \\
\hline 0 & $a_{0}$ & $b_{0}$ & $-a_{3}$ & $-b_{3}$ & 0 & 0 & & 0 & Thru (2) - Reverse \\
\hline
\end{tabular}

Fig. 2 : SOLT least sqare method

The second step of the measurement-setup calibration is the absolute calibration. If we calibrate the system according to a 12 error terms model [3], we have to find out the value of e10 in forward mode. The standard to get is a calibrated powermeter. A comparison between the powermeter value and the measurement-setup value of the incident power leads us to $\left|e_{10}\right|$ as described in [2]. The wave formalism used in this paper is defined as follow : $\mathrm{a}=\frac{\mathrm{V}^{+}}{\sqrt{\mathrm{Z}_{0}}}$ and $\mathrm{b}=\frac{\mathrm{V}^{-}}{\sqrt{\mathrm{Z}_{0}}}$ with $Z_{0}=50 \Omega$ [6]. Concerning the phase calibration, one assumes that the time group delay of one mixer (the phase reference one) remains constant in the measurement bandwidth. This bandwidth is small compared to the local oscillator frequency.

The presented measurement setup enables full envelope characterization of nonlinear devices at $\mathrm{L}$ and $\mathrm{S}$ band. Nevertheless, characterization at higher frequencies remains possible by the add of a second frequency translating stage as depicted in [7] for NPR measurements.

\section{MESUREMENTS OF THE NON LINEAR S PARAMETERS}

The setup was used to extract the scattering functions from a 2 stages HFET SSPA. The nonlinear scattering functions formalism, introduced by Verspecht [8], is limited here to the fundamental frequency but is extended to the accounting of high frequency memory effects as explained in [9]. Those nonlinear scattering parameters are defined as following :

$$
\begin{gathered}
\left(\begin{array}{l}
\mathrm{b}_{1}(\Omega) \\
\mathrm{b}_{2}(\Omega)
\end{array}\right)=\left[\begin{array}{ll}
\mathrm{S}_{11}\left(\Omega,\left|\mathrm{a}_{1}\right|\right) & \mathrm{S}_{12}\left(\Omega,\left|\mathrm{a}_{1}\right|\right) \\
\mathrm{S}_{21}\left(\Omega,\left|\mathrm{a}_{1}\right|\right) & \mathrm{S}_{22}\left(\Omega,\left|\mathrm{a}_{1}\right|\right)
\end{array}\right] \cdot\left(\begin{array}{c}
\mathrm{a}_{1}(\Omega) \\
\mathrm{a}_{2}(\Omega)
\end{array}\right) \\
+\mathrm{e}^{\mathrm{j} \cdot 2 \cdot \varphi\left(\mathrm{a}_{1}\right)} \cdot\left[\begin{array}{ll}
\mathrm{S}_{11}^{\Delta}\left(\Omega,\left|\mathrm{a}_{1}\right|\right) & \mathrm{S}_{12}^{\Delta}\left(\Omega,\left|\mathrm{a}_{1}\right|\right) \\
\mathrm{S}_{21}^{\Delta}\left(\Omega,\left|\mathrm{a}_{1}\right|\right) & \mathrm{S}_{22}^{\Delta}\left(\Omega,\left|\mathrm{a}_{1}\right|\right)
\end{array}\right] \cdot\left(\begin{array}{c}
\mathrm{a}_{1}^{*}(-\Omega) \\
\mathrm{a}_{2}^{*}(-\Omega)
\end{array}\right)
\end{gathered}
$$

Each scattering parameter is a function of the incident wave's magnitude and frequency.

If the measured waves are phase normalized with the phase of and one assume there is no low frequency memory effect in the device under test, one can simplify the relation
(2). Therefore the parameters and have least impact on the high frequency behaviors of the device and thus can be neglected in our context. It leads us to relation (3).

$$
\begin{array}{r}
\left(\begin{array}{l}
\mathrm{b}_{1}(\Omega) \\
\mathrm{b}_{2}(\Omega)
\end{array}\right)=\left[\begin{array}{ll}
\mathrm{S}_{11}\left(\Omega,\left|\mathrm{a}_{1}\right|\right) & \mathrm{S}_{12}\left(\Omega,\left|\mathrm{a}_{1}\right|\right) \\
\mathrm{S}_{21}\left(\Omega,\left|\mathrm{a}_{1}\right|\right) & \mathrm{S}_{22}\left(\Omega,\left|\mathrm{a}_{1}\right|\right)
\end{array}\right] \cdot\left(\begin{array}{c}
\mathrm{a}_{1}(\Omega) \\
\mathrm{a}_{2}(\Omega)
\end{array}\right) \\
+\left[\begin{array}{ll}
0 & \mathrm{~S}_{12}^{\Delta}\left(\Omega,\left|\mathrm{a}_{1}\right|\right) \\
0 & \mathrm{~S}_{22}^{\Delta}\left(\Omega,\left|\mathrm{a}_{1}\right|\right)
\end{array}\right] \cdot\left(\begin{array}{c}
\mathrm{a}_{1}^{*}(-\Omega) \\
\mathrm{a}_{2}^{*}(-\Omega)
\end{array}\right)
\end{array}
$$

As explained in [9], the pragmatic formalism (3) has been inspired by the Volterra theory. The basic idea was to expand the general laws governing a two port element using the dynamic Volterra series expansion. Considering a realistic use of an amplifier in terms of output impedance matching (practically, a bounded region around the nominal impedance defined by an reflection coefficient of about 0.3 ), the amplifier behaves linearly as to $\mathrm{a}_{2}$ and $\mathrm{a}_{2}^{*}$. For this reason, the Volterra expansion has been limited to the first order enabling both an accurate extraction of the [S] parameters using measurement setup and an efficient numerical implementation.

The set of 6 unknown scattering parameters are extracted from $\mathrm{CW}$ measurements. The incident wave is swept in power (low level to the DUT $3 \mathrm{~dB}$ compression gain) and frequency (40 Mhz around the center frequency : $1.6 \mathrm{GHz}$ ). Those measurements are carried out for three different loading impedances in order to solve the linear equation system (3). We have considered $\Gamma_{\text {Load }}=0,0.2 \mathrm{e}^{\mathrm{j} .0^{\circ}}$ and $0.2 \mathrm{e}^{\mathrm{j} .90^{\circ}}$. Some results examples are shown : AM/AM$\mathrm{AM} / \mathrm{PM}$ conversion for different frequencies $(\Omega)$ on figures 3 and 4 . "Hot S22" is presented on figures 5 to 8 . Basically, $\left|S_{22}^{\Delta}\right|$ hold out to small value for $\left|a_{1}\right|$ going to zero. This property is limited by the dynamic range of the receiver part.

\section{NUMERICAL IMPLEMENTATION OF THE VOLTERRA MODEL}

In order to be exploitable in a somewhat simulation environment, the formulation of the bilateral Volterra model (2) must be written in a more convenient shape. As in [10], the idea is to project the mapping of discrete points describing the parameters $\mathrm{S}_{\mathrm{ij}}\left(\left|\mathrm{a}_{1}\right|, \Omega\right)$ in a sub-space of continuous basic functions.

As it is often referenced in the literature [11], we have chosen to expand $\mathrm{S}_{\mathrm{ij}}\left(\left|\mathrm{a}_{1}\right|, \Omega\right)$ according to the incident power wave using monomials as basic functions. Doing that, we obtain :

$$
\mathrm{S}_{\mathrm{ij}}\left(\left|\mathrm{a}_{1}\right|, \Omega\right)=\sum_{\mathrm{k}=0}^{\mathrm{K}} \Psi_{\mathrm{ijk}}(\Omega) \cdot\left|\mathrm{a}_{1}\right|^{\mathrm{k}}
$$




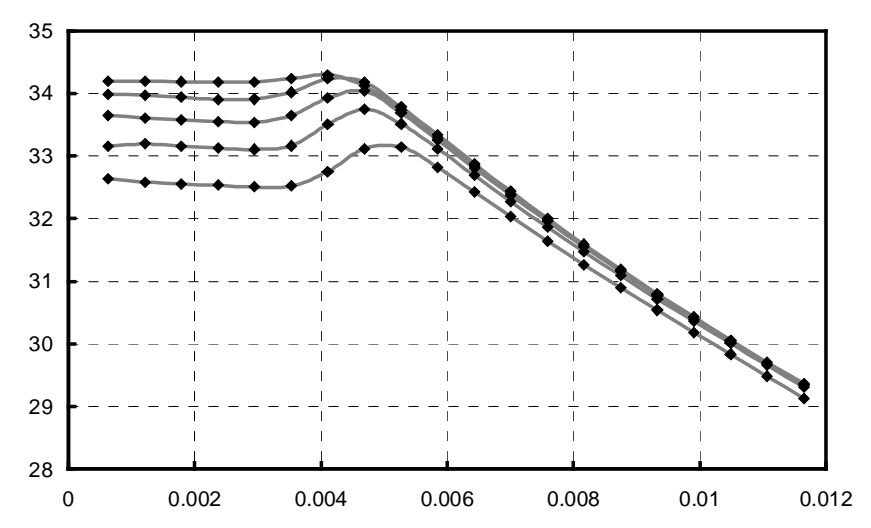

Fig. 3 : $\left|S_{21}\right|_{d B}$ versus $\left|a_{1}\right|$

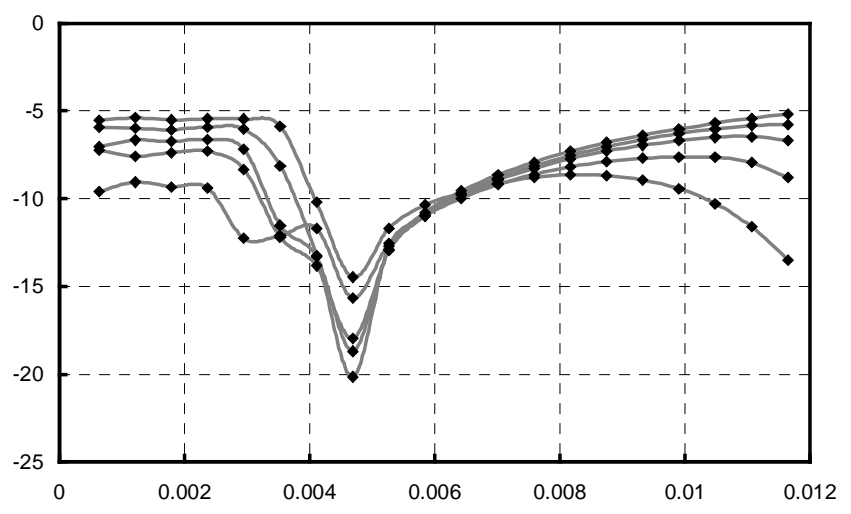

Fig. $5:\left|\mathrm{S}_{22}\right|_{\mathrm{dB}}$ versus $\left|\mathrm{a}_{1}\right|$

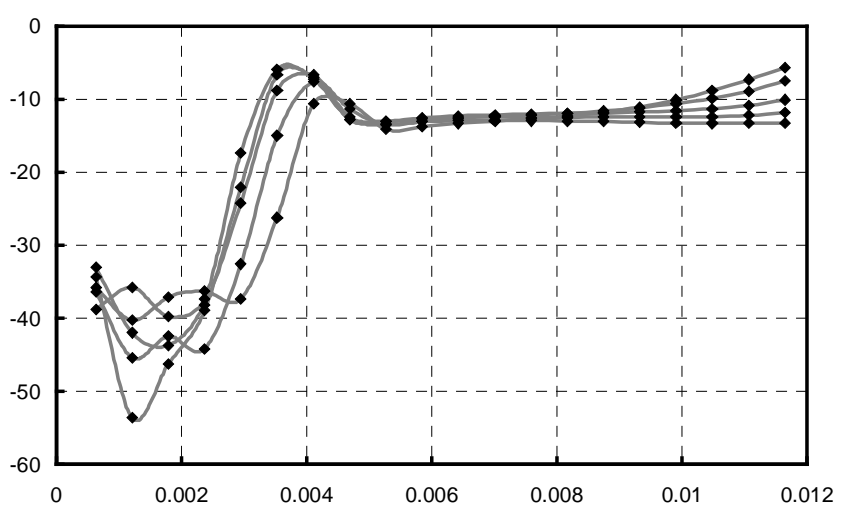

Fig. $7:\left|S_{22}^{\Delta}\right|_{d B}$ versus $\left|a_{1}\right|$

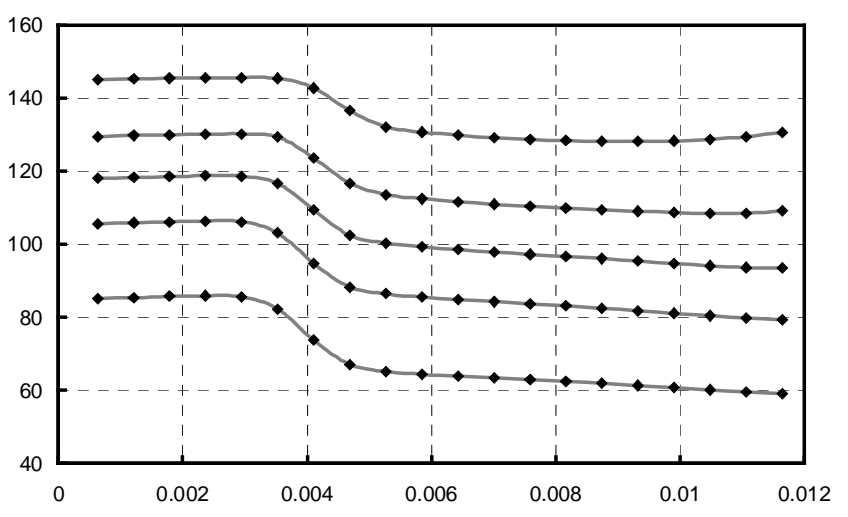

Fig. $4: \operatorname{Arg}\left\{S_{21}\right\}_{\circ}$ versus $\left|a_{1}\right|$

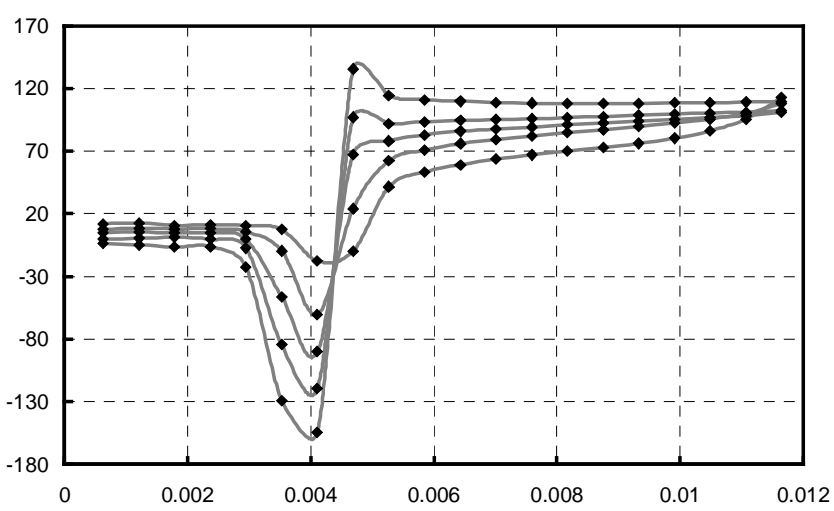

Fig. $6: \operatorname{Arg}\left\{S_{22}\right\}_{\circ}$ versus $\left|a_{1}\right|$

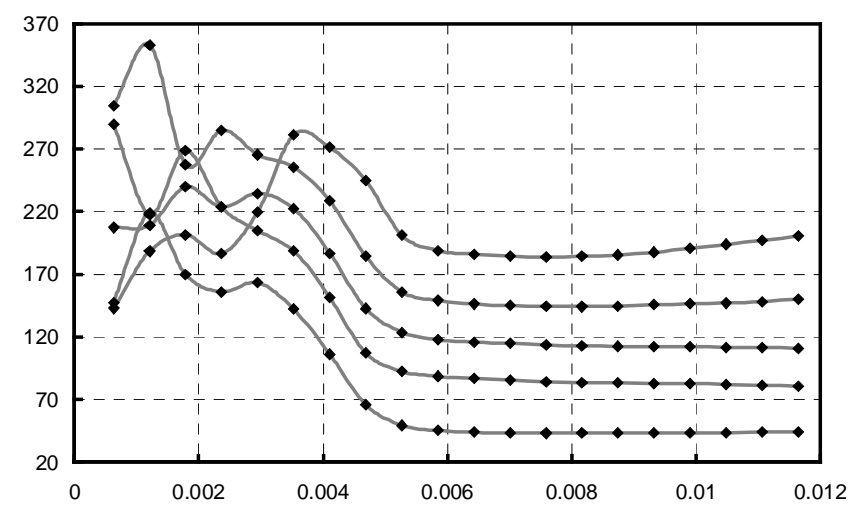

Fig. 8: $\operatorname{Arg}\left\{\mathrm{S}_{22}^{\Delta}\right\}$ versus $\left|\mathrm{a}_{1}\right|$ 
Using such a expansion, the contribution of each $\mathrm{S}$ parameter can be depicted as shown on figure 9 .

This equivalent network is thus well adapted for the usual circuit / system level simulation environment.

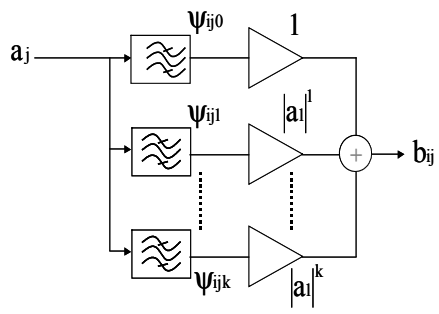

Fig. 9 : Synoptic of the Volterra model for one [S] parameter : Sij

Figures 10 and 11 present some simulations results with the measurement based Volterra model. Those figures show a comparison for 3 frequences and 2 different load impedance.

The simulations have been realized in Matlab/Simulink ${ }^{\mathrm{TM}}$ considering $\mathrm{K}=10$. Notice that the model fits a behavior depending on the frequency and the loading impedance. Phase mismatches displayed on the argument curves are introduced by numerical calculation problems. Those results on the argument of Vout may be upgraded by the use of another base functions for $\mathrm{a}_{1}$ in (3).

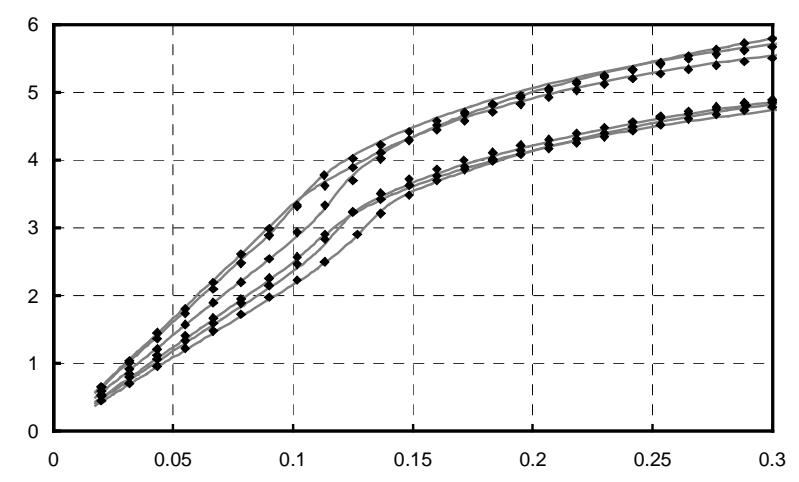

Fig. 10: $\left|\mathrm{V}_{\text {Out }}\right|_{\mathrm{V}}$ vs $\left|\mathrm{V}_{\text {In }}\right|_{\mathrm{V}}$. Simulation (curves) / Measure (dots)

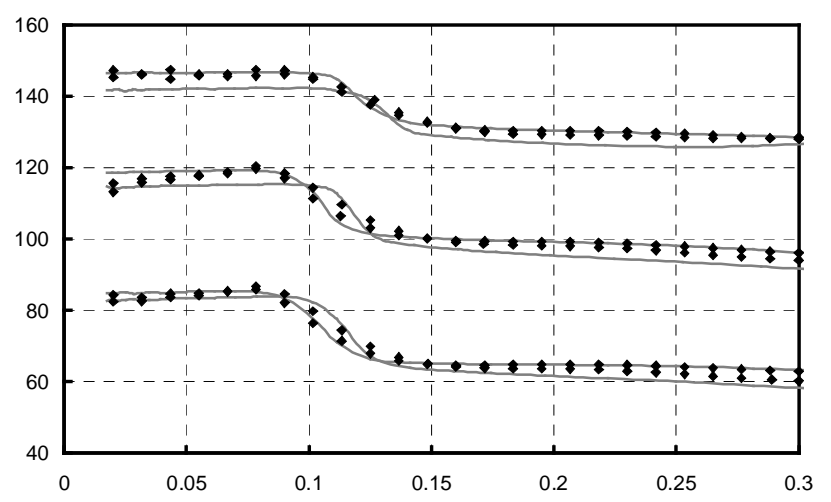

Fig. 11: $\operatorname{Arg}\left\{\mathrm{V}_{\text {Out }}\right\}_{\circ}$ vs $\left|\mathrm{V}_{\text {In }}\right|_{\mathrm{V}}$. Simulation (curves) / Measure (dots)

\section{CONCLUSION}

This paper has presented a new kind of characterization of nonlinear RF devices for complex envelope behavioral modeling. The setup principle focuses on complex enveloppe analysis dedicated to communication applications.

This mesurement tool can fully characterize fifty ohms matched ampliers in order to integrate system level envelope model in simulators. Nevertheless mismatche conditions are enabled both for testing and modelling of RF devices.

Then, a scattering parameter approach, taking into account nonlinearities within high frequency memory effect have been presented. Those measured non linear scattering parameters has been shown and used to build a model taking into account the output mismatch impedance.

\section{ACKNOWLEDGMENT}

The authors wish to acknowledge the TARGET organization for the financial support in the context of the Workpackage 2.2.B.1-NOE classic-B Front-End Sampler Group ( $\mathrm{n}^{\circ}$ contract : 507893).

\section{REFERENCES}

[1] T. Reveyrand, C. Mazière, J.M. Nébus, R.Quéré, A.Mallet, L. Lapierre, J. Sombrin, "A calibrated time domain Envelope measurement system for the behavioral modeling of power amplifier", EuMC 2002, Milan,GAAS, pp. 237-240, 2002

[2] J. Verspecht, "Calibration of a measurement system for high-frequency nonlinear devices", Doctoral Dissertation, Vrije Universiteit Brussel, Belgium, 1995

[3] D. Rytting, "VNA Error Models and Calibration Methods", NIST Short Course on Microwave Measurements and Instrumentation, Presented at the 62nd ARFTG, December 2003, Boulder, Colorado

[4] F. Purroy, L. Pradell, "New theorical analysis of the LRRM calibration technique for vector network analysers", IEEE Trans. on Instrumentation and Measurements, vol. 50, Issue 5, Oct. 2001, pp. $1307-1314$

[5] D.C. DeGroot, J. A. Jargon, R. B. Marks, "MultilineTRL Revealed", ARFTG Conference Digest, 60th, pp. 131-155

[6] J. A. Jargon, K.C. Gupta, D.C. DeGroot, "Nonlinear Large-signal Scattering Parameters : Theory and Applications", ARFTG Conference Digest, pp.157-174, June 2004

[7] A. Mallet, F. Gizard, T. Reveyrand, L. Lapierre, J. Sombrin, "A new satellite repeater amplifier characterization system for large bandwidth NPR and modulated signals measurements", IEEE MTT-S Digest, Seattle, Vol. 3 pp 2245-2248, June 2002

[8] J. Verspecht, "Scattering Functions for Nonlinear Behavioral Modeling in Frequency Domain", Fundamentals of Nonlinear Behavioral Modeling : Foundations and Applications workshop, IEEE MTT-S International Microwave Symposium, June 2003

[9] A. Soury, E. Ngoya, J. Rousset, "Behavioral Modeling of RF and microwave circuit blocs for hierarchical simulation of modern transceivers", IEEE MTT-S International Microwave Symposium, June 2005

[10] A. Soury, E. Ngoya, "Modeling of Long Term Memory Effects in Microwave Power Amplifiers for System Level Simulation", Annals of telecommunications, Accepted for publication.

[11] A. Leke, J. Stevenson Kenney, "Behavioral modeling of Narrowband Microwave Power Amplifiers with Applications in Simulating Spectral Regrowth", IEEE MTT-S International Microwave Symposium Digest, pp. 1385-1388, 1996 